\title{
Defining Haptic Experience: Foundations for Understanding, Communicating, and Evaluating $\mathrm{HX}$
}

\author{
Erin Kim, Oliver Schneider \\ Dept. of Psychology, Dept. of Management Sciences \\ University of Waterloo, Waterloo, Canada \\ erin.kim@uwaterloo.ca, oliver.schneider@uwaterloo.ca
}
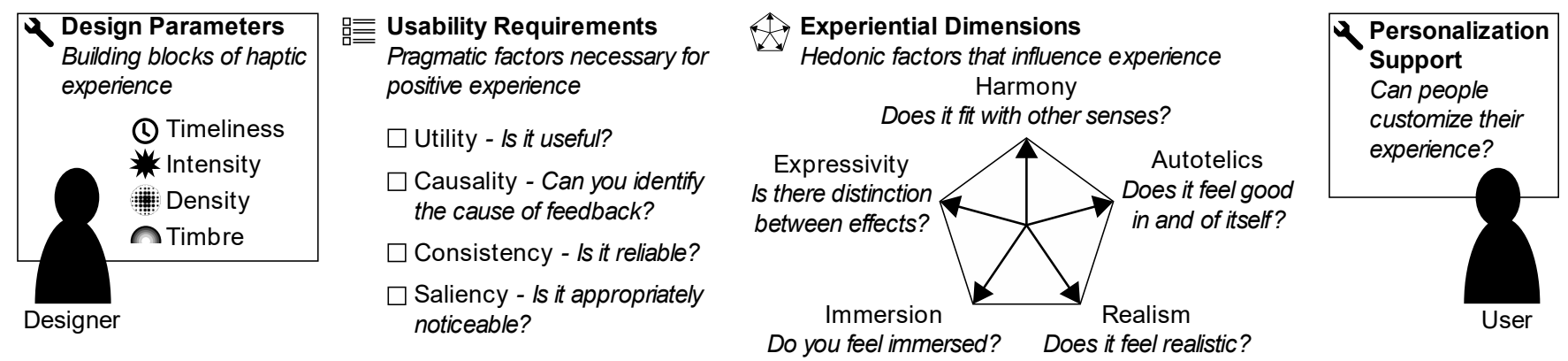

Figure 1 - The Haptic Experience (HX) Model: Designers of interactive systems can balance Design Parameters of Timeliness, Intensity, Density, and Timbre try to improve experience for people interacting with a haptic system. This will impact Usability Requirements, pragmatic factors important to focus on for haptics that also impact experience if not supported well: Utility, Causality, Consistency, and Saliency. We identify five Dimensions of HX that hapticians can target: Harmony, Expressivity, Autotelics, Immersion, and Realism.

\begin{abstract}
Haptic technology is maturing, with expectations and evidence that it will contribute to user experience (UX). However, we have very little understanding about how haptic technology can influence people's experience. Researchers and designers need a way to understand, communicate, and evaluate haptic technology's effect on UX. From a literature review and two studies - one with haptics novices, the other with expert hapticians - we developed a theoretical model of the factors that constitute a good haptic experience (HX). We define HX and propose its constituent factors: design parameters of Timeliness, Density, Intensity, and Timbre; the cross-cutting concern of Personalization; usability requirements of Utility, Causality, Consistency, and Saliency; and experiential factors of Harmony, Expressivity, Autotelics, Immersion, and Realism as guiding constructs important for haptic experience. This model will help guide design and research of haptic systems, inform language around haptics, and provide the basis for evaluative instruments, such as checklists, heuristics, or questionnaires.
\end{abstract}

\section{Author Keywords}

Haptics; User Experience; Design; Vibrotactile; Scale

Development

This is the author's version of the work. It is posted here by permission of ACM for your personal use. Not for redistribution. The definitive version was published in the Proceedings of the 2020 International Conference on Human Factors on Computing Systems on the ACM Digital Library.

\section{CSS Concepts}

- Human-centered computing Human computer interaction (HCI); Haptic devices; Interaction design theory, concepts and paradigms

\section{INTRODUCTION}

Haptic technology is becoming a widely available and increasingly expected part of user experience (UX). Companies like Apple and Nintendo have given developers access to high-fidelity inertial actuators in their devices, while startups like Tanvas and UltraLeap are adapting recent technologies like programmable friction $[3,55]$ and mid-air haptics [12] for consumers. The goal is often improved UX, with research evidence showing that physical props can increase presence in virtual environments [1], and haptic feedback can help videos be more pleasant, surprising, and novel [31].

Yet despite this promise, we have very little understanding of how haptic technology influences UX. Haptic designers, or hapticians [41], have developed their craft without strong theoretical underpinnings of haptic experience (HX). Without a firm understanding of the factors that constitute experience of haptic technology, hapticians will have limited means to articulate their design goals, elicit feedback from clients and stakeholders, and measure whether they achieve their goals. Research into haptic experience design (HaXD) has highlighted improved conceptual infrastructure, namely design languages and evaluative tools, as a key concern [41]. To improve this infrastructure, we need to understand what constitutes HX. 
In this paper, we propose the HX model, define HX, and identify the different factors that can influence experiences with haptic technology. From a literature review and two studies - one with haptics novices, the other with expert hapticians - we propose design parameters of Timeliness, Density, Intensity, and Timbre; usability requirements of Utility, Causality, Consistency, and Saliency; experiential factors of Harmony, Expressivity, Autotelics, Immersion, and Realism; and the cross-cutting concern of Personalization as guiding constructs important for HX.

The HX model will lead to improved understanding, communication, and evaluation with haptic technology. For example, when building a design, a haptician might tell their colleague that they feel it needs more Expressivity, more variation on the types of haptics the system can produce, and suggest adding more Timbres, the tones, textures, and colours of haptic feedback, to the design to remedy this. Once improved, the haptician might then bring it to a client, and use a questionnaire to measure how effectively the design supports the three HX goals they are trying to support: Expressivity, Harmony (the quality of multiple senses feeling integrated), and Autotelics (the haptic feedback simply feeling good in its own right).

In this paper, we contribute a definition for "haptic experience" accompanied by a model articulating the key elements constituting the user experience of haptic interactions. The model was derived from our studies: two workshops with eight haptic novices, and individual interviews with six expert hapticians. The model offers hapticians a common set of design targets, researchers constructs to consider with more nuance, and hapticians and users the vocabulary to more precisely communicate about HX. We conclude by discussing implications.

\section{DEFINING HAPTIC EXPERIENCE}

After conducting our studies and developing the HX model, we have developed a definition of haptic experience. We define haptic experience (HX) to be:

a distinct set of quality criteria combining usability requirements and experiential dimensions that are the most important considerations for people interacting with technology that involves one or more perceived senses of touch, possibly as part of a multisensory experience.

This definition combines the Sharp et al.'s description of UX [49] (informed by Schrepp et al's interpretations [43]) with Schneider et al.'s definition of HaXD [41]. We include "people" rather than "user" to be less gendered: participants in Bradley et al. [7] more often drew a male or masculine figure for "user" than not, suggesting it is a gendered term despite being expected to be neutral.

\section{RELATED WORK}

\section{Defining UX}

We adopt Sharp, Preece, and Rogers's definition of User Experience [49] as elaborated by Schrepp et al. [43]: "a set of distinct quality criteria that includes classical usability criteria, like efficiency, controllability or learnability, and non-goal directed or hedonic quality criteria, like stimulation, fun-of-use, novelty, emotions, or aesthetics." UX is largely understood as dynamic, contextual, and subjective, such that it is affected by a person's internal state (e.g. needs, motivations), dependent on the environment, and unique to each individual. Its subjectivity is impacted by the temporal nature of UX; given its applicability before, during, and after an interaction with a product $[2,30]$, UX can be shaped by an individual's past experience with a product or change even after the interaction concludes.

\section{Evaluating UX}

There exist several evaluation instruments outlining various features of UX. As reflected in its definition, UX is often characterized using two mostly-unrelated dimensions: hedonic and pragmatic, where hedonics is the perceived support for "be goals," such as being competent or related to others, and pragmatics is the perceived support for "dogoals," such as making a telephone call [21]. Hedonics reflects the aesthetics of a system [19], the non-instrumental components of a system. In contrast, pragmatics is more closely related to usability [20], and more to usage of the product or system.

Evaluation batteries, like AttrakDiff [19] or the User Experience Questionnaire (UEQ) [28] are organized around these two dimensions; the former has been used to measure the added value of haptics [31]. AttrakDiff measures users' perceptions of a product's pragmatic (e.g. controllable) and hedonic (e.g. innovation) qualities and how they contribute to the assessment of the overall attractiveness of the product [52]. The UEQ offers six subscales measuring the pragmatic qualities of perspicuity (learnability of a product), efficiency (ability to complete tasks with ease), and dependability ( user's sense of control over the interaction), and the hedonic qualities of stimulation (excitement and motivation produced from product use) and novelty (creativity of a product) [28]. Similar to AttrakDiff, the UEQ also evaluates the product's attractiveness (the overall impression of the product), under which pragmatic and hedonic qualities are subsumed. The Components of User Experience (CUE) model, evaluated with the meCUE questionnaire [32], is categorized in a similar fashion using the terms instrumental and noninstrumental qualities. Instrumental qualities parallel pragmatics, for example, referring to the system's usability, whereas non-instrumental qualities equate to hedonic elements, such as the system's look and feel. The CUE model suggests the two components of UX hold a bidirectional relationship with the third component: the user's emotional reaction to the product, reflective of the quality of attractiveness in the previous models. 


\section{The Experience of Touch}

While much research into haptic technology has focused on utility for specific tasks like training motor skills [16], several researchers have looked into emotional properties and perceptual dimensions of touch, some of which has translated to haptic technology.

Explorations of the experience can be difficult because the language surrounding haptic technology is difficult to define $[25,41]$. Still, studying this language has revealed useful insights. Research into how people talk about tactile experiences suggests mid-air tactile feedback [12] can mediate emotions through location on the hand and other physical parameters [35] in addition to descriptions of physical phenomena [36]. When we feel something we get an immediate sense of what we like or don't like, but often find it difficult to describe touch beyond that [25]. This has been shown to generalize to vibrotactile feedback [42].

While the language of touch is difficult to define, some taxonomies of touch have been created. Perception of materials and textures, for example, has evidence for five dimensions: hardness (hard/soft), friction (moist/dry, sticky/slippery), fine roughness (rough/smooth), macro roughness (uneven, relief), and warmness (hot/cold) [37].

We also understand some factors that influence the aesthetics of touch. Object properties can influence the "touchability" of an object for hedonic purposes, when they have intermediate levels of complexity, both of texture and shape [27]. Dimensions of texture, (e.g., micro/macro roughness, hardness, friction) have also been linked to texture [37], and even been captured in a computational model as a Bayesian network [33].

Sound-symbolic language like onomatopoeia also play a role when describing touch. Tactile parameters (e.g., smooth/rough, bumpy/flat) can be visualized [53] and predicted [15] from people's descriptions using soundsymbolic language (i.e., onomatopoeia like "zara-zara"). Designers also use onomatopoeia to express vibrotactile sensations (vibrations felt in the skin) [42].

The feeling of touch can be highly individual and context dependent. For example, dimensional spaces can have different numbers of dimensions depending on the individual [23], and vibrotactile feedback can depend on body location and current activity (e.g., walking vs. standing) [26]. Some people are more or less likely to want to touch for autotelic purposes, i.e., hedonic touch for its own sake [25, 38]. Hapticians have described haptic sensation as being "very individual" [41].

Personalization is thus critical for supporting touch-based interfaces [45]. Affective responses to vibrations can vary according to individual differences in demographics, use, and tactile processing capabilities [47]. The way that people make sense of haptic feedback can be organized into different haptic facets [48], categories of attributes that characterize collections of sensations. Five facets for vibrotactile feedback have been compiled [46], suggesting that people can frame vibration feedback using physical, sensory, emotional, metaphoric, or usage attributes.

While not purely experiential, affect clearly plays a role in touch [25] and, unsurprisingly, with haptic technology. Early explorations of haptic technology like InTouch [8] proposed using haptics to facilitate interpersonal communication, leading to natural and emotional communication. Work into social haptic robotics involving haptics [57] has found ways to both influence emotion (relaxing the user) through physical feeling of breathing [44] and been successful at classifying gestures of touch input $[11,17]$ by their intended emotional state. Visual robot emotion perception of this sort changes based on narrative framing [10]. Affect has also informed design of vibrotactile icons, linking physical parameters like amplitude and frequency to emotional responses [58]. Affective response can depend on multimodal effects: a crossmodal design, e.g., with visual and haptic components, depends on the agreement of its two component parts [59], and there is an "uncanny valley" of haptics, where spatial haptic feedback with no visual feedback had lower immersion than either generic haptic feedback or spatial haptic and visual feedback [4].

While affect has become a common target, more general support of HX is rare. Systems designers of haptic systems have proposed taxonomies for supporting Quality of Experience (QoE) for haptic technology [18]. QoE is typically a measure of the experience of a service, here, of haptics delivered to a user. This model separates Quality of Service (QoS) parameters, such as response time, latency/delay, price, reliability, from UX parameters proposed as perceptual qualities, rendering quality, physiological measures, and psychological measures. This overall taxonomy includes a large number of parameters, but needs elaboration and focus to guide designers or result in a practical evaluation questionnaire.

Researchers have also linked the addition of haptic feedback to some possible experiential goals. Adding haptic feedback can improve UX: adding mid-air haptics and vibrotactile feedback to videos resulted in more pleasant, unpredictable, and creative experiences [31]. The use of haptic feedback can increase immersion and presence, two constructs frequently targeted in VR and telepresence systems [1], and used as proxies for measuring $\mathrm{HX}$.

\section{Related Constructs and their Scales}

Experience designers and hapticians use several constructs related to $\mathrm{HX}$ as design goals, and can evaluate those constructs with scales or relate them to user traits. The positive and negative affect schedule (PANAS) measures positive and negative affect [13] and features an extended form [54]. Russell's circumplex model of affect [40] identifies constructs of valence (positive/negative affect) and arousal (excitation), and is frequently used in affective design involving haptics [58]. 
Virtual environments often employ presence, the subjective experience of being in one place or environment, even when one is physically situated in another [50]. Researchers can employ the Presence Questionnaire [56] to measure presence and related concepts, such as immersion, including supporting factors like involvement and immersion. This has been used to study haptics in VR, e.g., with passive haptics [1]. Others have used immersion as their preferred construct for subjective experience [4].

Games user researchers are actively developing means to evaluate experience. We reviewed constructs in the Gaming Engagement Questionnaire (GEQ) [9], but are aware that it has not been validated [29]. We drew more from the Player Experience Index (PXI), which received input from 64 experts for its initial design and development. The PXI measures Enjoyment, which is composed of two collections of factors: Psychosocial consequences (Aesthetics) involving Mastery, Curiosity, Immersion, Autonomy, and Meaning; and Functional consequences (Dynamics), involving ease of control, progress feedback, audiovisual appeal, clarity of goals, and challenge. Models of player traits attempt to manage individual differences in this domain as well [51]. We drew upon similar scale development processes to the PXI in order to generate ideas for what might be important for HX, namely, by engaging novices and experts.

\section{Differentiating $\mathrm{HX}$ from UX}

We believe that designers and researchers will benefit from a focused definition of HX rather than following existing UX models. While the elements offered by existing UX models remain relevant to $\mathrm{HX}$, there are key components unique to haptic interactions that have yet to be captured. For instance, a haptic experience is highly dependent on the interplay between physical feedback and other components of a system, as well as other senses and contextual factors. Haptics is highly multi-modal, demanding that hedonics and pragmatics be defined differently. Another example: HX differs from UX in its time frame. While UX applies to the time before, during, and after an interaction, HX depends highly on focused interaction in the moment.

Differences like these suggest that different constructs should be prioritized when designing for haptic technology. We thus set out to find out what constructs are important.

\section{APPROACH: SCALE DEVELOPMENT}

Our original goal was to create a questionnaire measuring HX. However, the process of developing the scale revealed the need to identify and define HX and discover its key constituents; without this key prerequisite, we are left without guidance as to what to measure. Research into HaXD [41] highlights a need for conceptual infrastructure, including evaluation methods for haptic systems. As no prior work had established criteria with sufficient detail to determine whether a haptic experience can be evaluated as successful, we shifted our focus to defining $\mathrm{HX}$, leading to the creation of the HX model. Similar to how early theoretical work defined the construct of presence [50] and led to the Presence
Questionnaire [56], the HX model can now serve as a foundation on which long-term goals, such as a future HX questionnaire, can be developed. Researchers and designers could use such a questionnaire to evaluate their systems and develop principled guidelines for haptic systems, much like how the Presence Questionnaire has guided work in VR and telepresence.

We begin the process of scale development to identify the constructs that make up the HX model. The initial steps of scale development involves both novices and experts to develop and refine the constructs to be measure $[6,14]$. We began by reviewing prior research and input from novices and experts. We reviewed existing taxonomies for haptics, user experience, VR and gaming. We then invited eight novices to brainstorm what elements constituted a good haptic experience. We developed 50 questionnaire items from the brainstormed elements. We then interviewed six expert hapticians about their evaluation techniques and asked them to perform a card sorting task with these items.

The result, at this stage in the process, is the identification of what constructs make up HX: the HX model. We developed this from literature on haptics and user experience, input from novices, and interviews with expert hapticians.

\section{STUDY 1: NOVICE BRAINSTORM}

A critical aspect of developing the construct of HX is to include the people for which this construct matters, i.e., users of haptic systems. While knowledgeable hapticians can use their expertise to inform the development of the HX construct, the observations of those with limited haptic experience can offer unbiased, bottom-up insight, also valuable to understanding haptic interactions. Therefore, haptic novices were recruited to begin the process of construct development. We held two workshops with eight people (four people per workshop) to provide an opportunity to interact with a haptic system and speak to the experience. The purpose of the sessions was to brainstorm what constitutes good HX, thereby allowing us to begin compiling a concrete set of ideal experience elements that direct the criteria aimed for by hapticians and expected by users.

\section{Choice of Haptic Device: Nintendo Switch}

While our long-term goal was to create a model generalizable to all haptic technology, we started with a specific case. We chose the Nintendo Switch as our example device because it renders a commonly implemented haptic modality (vibrotactile feedback). This console hosts high fidelity inertial actuators, which has led to haptic feedback being specifically praised for some games [5, 24, 34].

\section{Participants}

We recruited eight haptic novices through our personal and professional networks to include a diverse group of people with limited familiarity with haptic technology. Eligibility criteria included individuals between the age of 18 to 64 years and those who have not studied or worked in the field of haptics. In each workshop, indicated by self-reported 
gender, were two male and two female participants. The age ranged from 21 to 28 years in the first workshop, and 21 to 35 years in the second workshop. Across both groups, there was variation in participants' field of study, including fine arts, psychology, engineering, and computer science, as well as variation in experience playing video games.
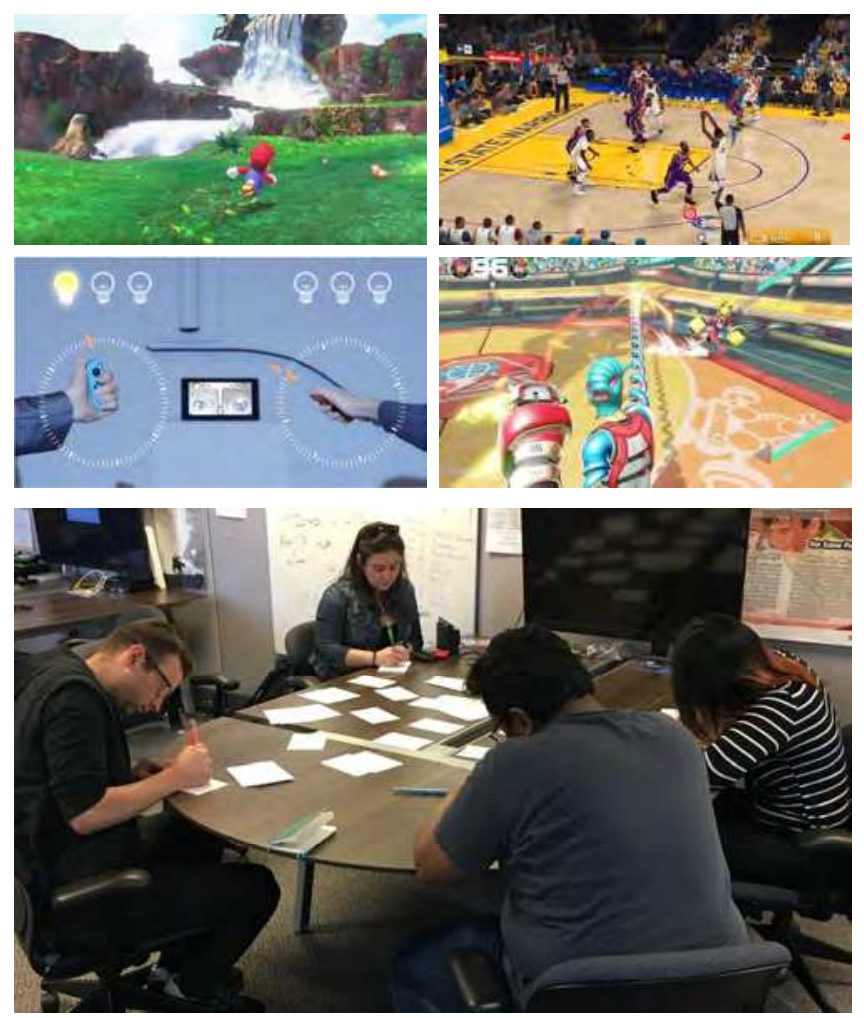

Figure 2 - Novice brainstorm (first of two sessions) and selected Nintendo Switch games. Top: Games included; clockwise from top-left: Super Mario Odyssey, 2 K19 NBA, Arms, and 1-2Switch. (Bottom) Brainstorming participants. Participants were given a presentation about haptic technology, brainstormed factors affecting haptic experience, played several games on the Nintendo Switch that involved a variety of vibrotactile feedback, then revisited their brainstormed factors, and finally voted on the most important factors.

\section{Procedure}

The session began with a brief presentation to familiarize participants to haptic technology and its various applications. We highlighted the objective of developing an evaluation questionnaire and described the goal of the session: to identify valuable elements of haptic experiences to be captured in the scale. At the end of the presentation, participants were given five minutes to independently write ideas on cue cards in response to the question, "What constitutes a good haptic experience?" We asked this question to begin selecting the elements of haptic interactions that should be included in the questionnaire; that is, elements that were desirable and perceivable to users. Suggestions were then presented one at a time and clustered together based on related concepts.
A second brainstorm period followed. Participants played four games on the Nintendo Switch: Super Mario Odyssey, $2 K 19 N B A, 1-2-S w i t c h$, and Arms. To offer a range of experiences, the games were selected based on the variation of haptic intensity, diversity of effects, and necessity of the haptics to complete the player's objective. For example, the 1-2-Switch game Safe Crack required full dependency on haptic feedback to open a safe. Players rotated their controllers until they felt a more salient (louder) haptic effect, indicating the correct number for combination. This contrasted 2K19 NBA which used haptics to reflect a player's movement (e.g., shooting the ball) but was not at all required to execute the move. Participants rotated through the chosen games on two consoles, playing in pairs. All participants were required to play a set number of rounds or complete a pre-determined list of tasks in each game to ensure all players experienced similar haptic feedback.

Once all games had been tested, participants were again asked to brainstorm ideas for the previous question, "What constitutes a good haptic experience?" Additional ideas were expected to be created as participants now had first-hand experience interacting with a haptic device. New or refined suggestions were presented and added to the existing pool. Upon finalizing the factors, participants were asked to vote for those they considered the most important towards creating a good haptic experience. Each participant was given three votes.

\section{Results}

The novice brainstorming sessions resulted in ten initial factors describing what constitutes a positive haptic experience (Figure 3). Four factors significantly overlapped between the two novice groups; in these cases, factors were amalgamated and original labels were combined. For example, both groups listed similar ideas that reflected the importance of haptic effect timeliness; the first group assigning the label Response Time and the second group using the name Immediate Feedback. These two factor groups were combined in the final results. Novice contributions that considered only the industrial design and ergonomics of the game controller itself, rather than its associated haptic effects, were removed. The final factors formed the basis of which a collection of scale items was constructed, following instructions of item generation [14].

\section{STUDY 2: EXPERT REVIEW}

The next step in scale development was to have experts in the field evaluate the items for relevance, representativeness, and clarity $[6,14]$. Presenting the items for critique provided hapticians with the opportunity to challenge the ideas put forth by haptic novices, reword factor labels with more representative terms, specify unfocused items requiring revision, and offer recommendations for where there existed gaps in the constructs. 


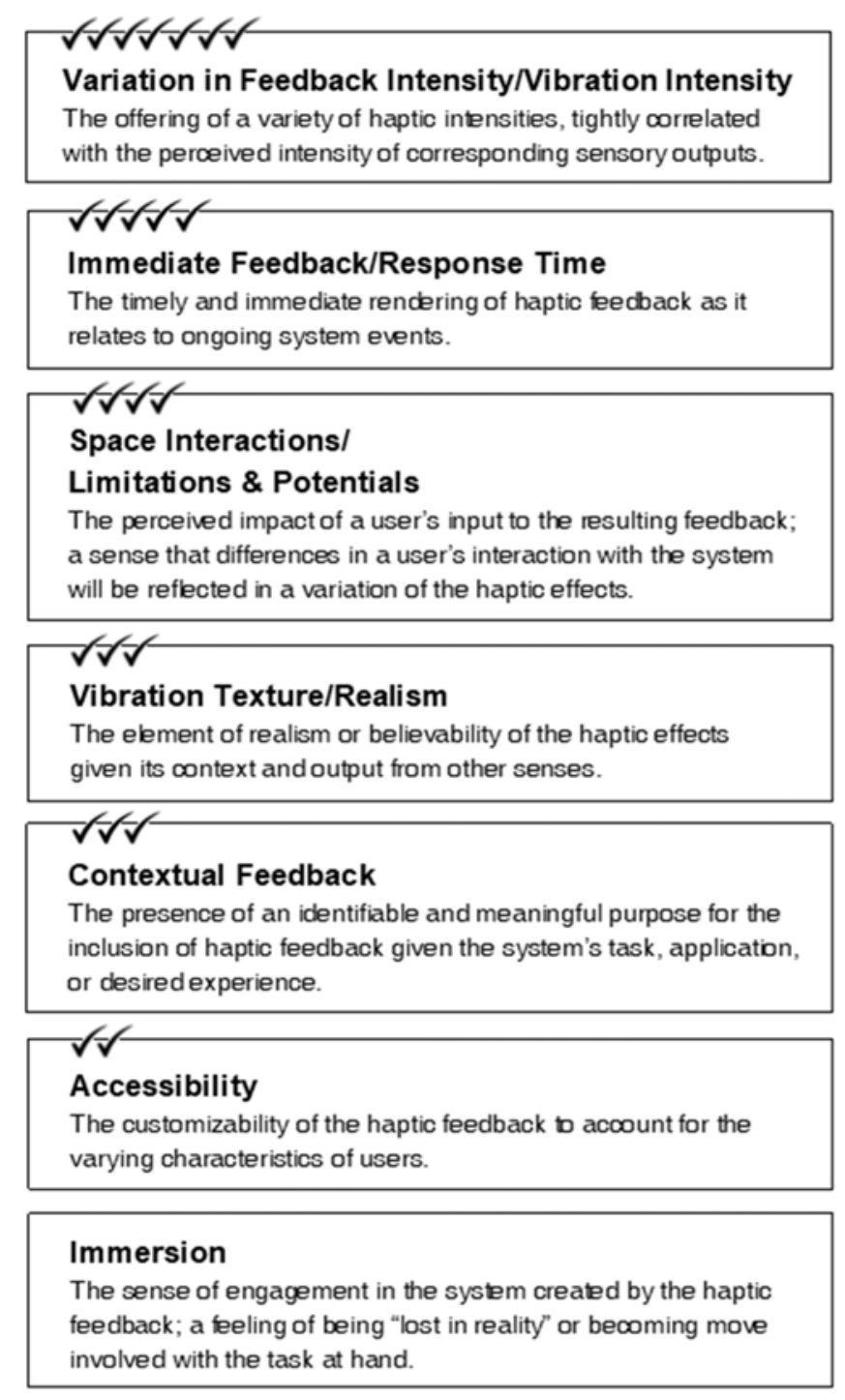

\section{Appropriate Frequency}

The rendering of a quantity of haptic effects within a given window of time appropriate for its context or purpose, conveying a cesired level of detail.

\section{Combining the Senses}

A tight alignment between the haptics and input of other sensory modalities, bebw the level of the system application.

\section{Ease/Learnability}

The intuitiveness of the haptics; an ease in understanding what the feedback aims to communicate to the user.

Figure 3 - Novice responses to the question, what makes a good haptic experience? Overlapping results between the two groups were combined and original factor labels are listed. $\checkmark$ represents one vote from novices denoting elements considered most valuable to HX. Novices greatly valued the effect variation, system responsiveness, multi-modal alignment, usefulness, and customizability.

\section{Participants}

We recruited six haptic experts (HE1-6). Experts were selected to achieve diversity in the following criteria within the group: age, gender, current career domain (i.e., industry vs academia), haptic modality experience, and when possible, location. All demographics were self-reported. The age of experts ranged from 32 to 53 years. Four experts selfidentified as male, and two experts self-identified as female. HE1-3 work in industry, while HE4-6 work in academia. There was a variance in the types of haptics used in their work, some of which included vibrotactile, force feedback, and inertial haptics. Multiple experts noted working with several haptic modalities simultaneously within their projects.

\section{Procedure}

Experts were individually invited to participate in an interview and card-sort task to further inform and organize the factors of HX. The interview was conducted to ask experts to describe currently used evaluation methods and any notable barriers faced in the process. We then described the purpose of the developing scale and the steps completed with the haptic novices to provide experts with relevant background necessary for the card sort. Experts completed the open card sort task online using OptimalSort (Figure 4). 50 items (five items per factor) had been generated, from which experts were to group items together based on criteria they felt was most appropriate and assign labels to each cluster. Follow-up questions were asked to receive feedback on the perceived relevance and clarity of items, and suggestions on important concepts absent from the draft that should be considered when evaluating haptic experiences.

\section{Interview Results}

The interviews with experts resulted in two main findings: a description of current evaluation methods and a set of barriers to evaluation.

\section{Result 1: Current evaluation methods}

Expert hapticians were asked how they have previously evaluated the haptic portion of their systems. When discussing user experience evaluation, some methods were mentioned without further comment (i.e. user studies, analytics), and three methods were elaborated: focus groups, administration of self-developed surveys, and the removal of haptic feedback.

HE1 used focus groups to present a set of haptic effects and request users to arrange them in order of desirability. Interestingly, focus groups had only been mentioned by one participant. Perhaps the difficulty of communicating about haptic experiences lead focus groups to be seen as demanding on the part of the user for sharing insight.

Surveys were mentioned by three experts: HE1 used a survey focusing on how haptics were being used in their devices; HE3 administered a self-developed scale exploring experiential themes including multi-modal alignment, realism, comfort, and satisfaction; HE6 used a scale asking users about their sense of immersion in a haptics vs non- 
haptics scenario. HE3 stated that they had to create their own questionnaire after being unsuccessful in finding a similar tool in literature. Similarly, HE6 considered using alternative scales such as the NASA-TLX questionnaire but found that many of the items were not directly applicable to experiences offered by haptic systems. They stated that "it would be great to have something similar that focuses on our needs" (HE6). The focus area of each survey differed across experts and two hapticians pointed to the discrepancy between what they require in a scale to measure haptic systems and what is currently available in literature.

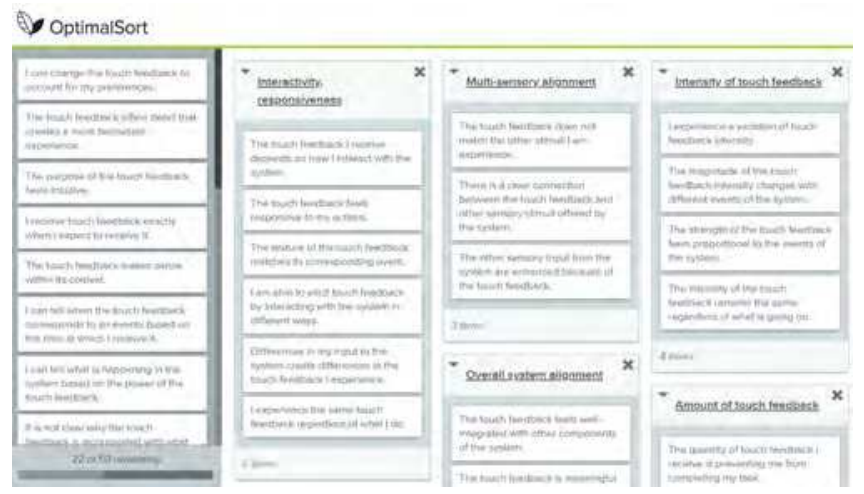

Figure 4 - Open card sort task using OptimalSort. Hapticians were to organize the items generated from novice factors into as many groups desired using the criteria they best saw fit.

Another common evaluation method across experts included presenting their systems without the haptic component to evaluate the effect on user performance and perceived system value. As was stated, "we know we're in a sweet spot when we take [the haptics] back, people feel they're really missing something" (HE2). Similarly, HE6 noted the usefulness of the designed haptics can be seen if the inclusion of haptics increased precision or reduced the time required to complete a task. The value of implementing haptics could be vouched for through finding discrepancies in task performance or system enjoyability.

HE1 and HE2 also took a designer-centered evaluation approach. While both experts seek out and consider the feedback received by end-users, they also rely on the sense of a designer, be it themselves or another party. HE2 offered their system to a diverse population of haptic and non-haptic designers and engineers. They also evaluated them personally, trying them directly and tuning various parts and seeing if reactions change. HE1 looks to a senior designer with experience and vision to provide the mark of approval. They saw the value of a designer-centered approach, saying "we need opinionated designers who have the track record of success that allows people to refer to them for these matters and believe that their opinion is relevant" (HE1). Both userand designer-centered were welcomed and deemed valuable by hapticians.

\section{Result 2: Barriers to Evaluation}

Being aware of the absence of a standard, widely available evaluation tool, hapticians were then asked if they had previously come across any barriers when selecting an evaluation method. HE5 and HE6 highlighted the dilemma of methods noticeably differing between hapticians. HE5 said, "it would be great to have a consistent tool because I think everybody at the moment is building their own approach." This hinders the ability to make comparisons between haptic systems, as was noted by HE6: "it's very hard to compare your haptic feedback, your haptic device, with others because of course when everybody uses their own method to evaluate, it's very hard." When offered the idea of a user experience scale for haptic technology, four experts (HE3-6) affirmed the creation of a universal tool that could be applied to multiple systems, thereby reducing independent labor and enabling comparative assessments.

As previously discussed, experts commonly removed the haptic component altogether to assess its value through its absence. However, HE5 mentioned that novelty often corrupted the data when evaluating using a non-haptic version of the system, particularly for those who are less experienced with haptics. The same expert noted it would be useful for the evaluation tool to be able to account for the discrepancy and transition between novice and expert users given that feedback will vary based on the level of experience.

HE1 spoke to the difficulty of eliciting language directed towards haptic experiences: "A lot of people don't know what haptics is, don't know to expect it, so you are challenged to get people to articulate in ways that are useful to you" (HE1). Lacking accessible language for users to describe haptics, particularly those with little experience interacting with haptics, have left evaluators to receive unfocused feedback. Having a concrete set of criteria to form the base of haptic evaluation can assist both designers in probing the desired feedback and haptic novices in communicating about their experiences.

Additionally, haptic experiences are challenging to share and, therefore, difficult to evaluate remotely. HE4 stated that those in the role of a reviewer can find it challenging to have a sense of the HX a system offers when it is not available for direct use. When asked how they would use a universal haptic evaluation tool, HE4 stated they would use it as way have hapticians prove their systems offer good HX.

\section{THE HX MODEL: FACTORS FOR HAPTIC EXPERIENCE}

From the literature, brainstorming sessions with haptic novices, and interviews with expert hapticians, we developed the HX model for Haptic Experience (Figure 1). This model links controllable Design Parameters (Figure 5), and Personalization Support to Usability Factors (pragmatics of HX, Figure 6) and Experiential Dimensions (hedonics of HX, Figure 7). 


\section{Card Sort Results}

To create the elements of the model, the categories of items formed by the experts were sorted based on similarity and labelled to describe the theme; some were named using terms established in literature (e.g. immersion), offered by experts (e.g. harmony), or discussed in novice sessions (e.g. saliency). Experts varied in the specificity of their categories; some provided broader categories that subsumed several concepts, others produced more categories that were more granular in scope. With the aim to inform design and generate a vocabulary for $\mathrm{HX}$, greater specificity was chosen for the model. As a result, where applicable, categories were divided to be more focused. For example, HE5 offered a category describing the appropriateness of haptic feedback which, based on their description, could be further divided to eventually form the elements of timeliness and harmony. Furthermore, some suggested elements were not included in the final model. HE1, HE3, and HE4 discussed the element of intuitiveness, the user's ease of understanding the purpose of haptic effects; however, this element was not included in the model as it not informative for design purposes. As stated by $\mathrm{HE} 1$, rather than designing for intuitiveness, a more attainable goal is to design multi-modal experiences that make sense. Drawbacks was another eliminated category as it involved the negative components of HX. The items that formed this category still addressed in the model, simply inversely in a positive light. For example, the concern for believability in haptics in the item "The touch feedback does not reflect reality," is captured in the element of realism.

Design Parameters
Building Blocks of Haptic Experiences
The factors that hapticians can directly manipulate to
influence the experience of a haptic system.
Timeliness: temporal alignment with other sensory
outputs or prompt response to system events and input.
Intensity: the overall perceived strength of feedback.
There should be a clear relationship between the magni-
tude of the haptics and the purpose of the feedback.
Density: the rate of haptic feedback, i.e., the amount of
haptic effects produced within a given time. Similar to
intensity, the density of feedback should align with the
intention behind its inclusion.
Timbre: the overall tone, texture, colour, or quality of the
feedback. Independent of the previous control parame-
ters, timbre isolates the character of the effect itself.

Figure 5 - Design Parameters that hapticians have direct control over. These are designed to be abstract enough to deal with different types of haptic feedback

\section{Design Parameters: Building Blocks of $\mathrm{HX}$}

Design Parameters are aspects of the HX that are tunable by hapticians and, in some cases, users. These are general building blocks used across diverse haptic devices and adjusted to influence HX. Design Parameters include Timeliness, Intensity, Density, and Timbre. The term Density was suggested by HE1 to describe the number of haptic events within a certain period of time. Both HE1 and HE3 separated the quantity of feedback from other factors, initially using the labels "Pervasiveness" and "Amount of Touch Feedback", respectively; "Density" was later suggested.

Usability Requirements
Pragmatic factors necessary for positive experience
While these are important in usability generally, they are
the focus when designing for haptic technology.
Utility - Is it useful?
The ability of haptics to benefit the user experience in
ways other sensory modalities cannot.
Causality - Can you identify the cause of feedback?
Causality describes how easily a user can relate haptic
feedback to the source of interaction.
Consistency - Is it reliable?
The system's ability to provide the same haptic feedback
across identical system inputs and events.
Saliency - Is it appropriately noticeable?
The noticeability of the haptic feedback as it relates to its
purpose and context.

Figure 6 - Usability requirements, pragmatic factors of $\mathrm{HX}$ important for usability with haptics. If these are not supported, they may have adverse effects on experience.

Usability Requirements: Pragmatic Factors of HX

The usability requirements describe aspects of functionality in HX including Utility, Causality, Consistency, and Saliency. The fulfillment of these requirements creates practical value in haptic systems below the level of a hedonic experience, and had been described by experts as factors that must be fulfilled before building upon the experience. For instance, Consistency was described as a technical requirement, "a given that you would want the haptics to be consistent" (HE1). Causality was also defined as a factor where, if not satisfied, can disrupt the ability to build an experience: "If it's a mismatch from [Causality], I think it's kind of hard to build on top of it" (HE2). Haptic systems should be designed in a way that allow users to intuitively understand what caused the feedback they received.

Utility addresses the value of haptics in serving as an additional channel through which information can be transferred to users. Unique in being eyes-free and a highly personal method of communication, haptics can provide users with insight into system events above and beyond that of alternative senses. Utility can be qualified by either enhancing user performance (e.g. touch feedback used to inform a player of their avatar's surroundings in Super Mario Odyssey) or enabling skills that would not otherwise be available without touch interactions (e.g. permitting players to determine the combination to open the safe in Safe Crack).

Saliency is characterized by the noticeability of feedback to users. Haptic effects should capture or shift users' attention to 
a degree that is suitable for its purpose. If the haptics are too subtle, they may go unnoticed, rendering them ineffective. Conversely, they may feel too aggressive or pervasive, becoming distracting or annoying to the user.

Experiential Dimensions
Hedonic factors that influence experience
Harmony - Does it fit with the other senses?
The most important dimension; how tightly coupled the
haptics are perceived to be with other sensory outputs.
Autotelics - Does it feel good in and of itself?
A positive haptic experience can be partially achieved
through the touch feedback feeling pleasant, independent
of its purpose in the system.
Expressivity - Is there distinction between haptic effects?
Expressive haptics are those that distinguishably reflect
varying user input and system events. Expressivity allows
users to feel their input makes an impact on the feedback
received.
Immersion - Do you feel immersed?
Immersion focuses on the level of engagement in the
system or application the user feels as a result of experi-
encing haptic feedback.
Realism - Does it feel realistic?
Whether the haptic effect convincingly portrays what
someone would expect to feel in reality.

Figure 7 - Experiential dimensions; hedonic factors of $\mathrm{HX}$ that hapticians can target when trying to create a positive haptic experience.

\section{Experiential Dimensions: Hedonic Factors of $\mathrm{HX}$}

The experiential dimensions represent the hedonic factors of HX, constituted by Harmony, Autotelics, Expressivity, Immersion, and Realism.

Harmony was a theme that arose in all results of expert card sort tasks and was indicated multiple times as one of the most important themes for HX. Experts stated that even if you have a very simple or limited kind of haptic feedback, it can still be meaningful if well integrated with the other modalities offered by the system. HE6 stated "at the end of the day, even if you have a very simple haptic feedback, it's very important it fits very well with the other senses...if you have a very advanced haptic interaction but you don't have good matching with what you're showing with the other senses, it won't work". Previous research has provided the similar term congruence, defined as an "intuitive match or harmony between the designs of feedback from different modalities" [22]. Our work supports its findings that congruent cross-modal effects leads to higher perceived quality. The term harmony, used by HE2, will continued to be used in the HX model to differentiate from congruence, specifically to capture experts' suggestion that harmony in and of itself can create a meaningful experience.

Realism can be a product of achieving harmony should it align with the designer's intention. However, it can also be achieved independent of other sensory outputs. This is can be seen in a system rendering electrovibration technology, which can produce a texture that feels realistic in isolation or in relation to an associated visual stimulus.

Immersion can also be produced through attaining harmony if desired for the system. Creating a sense of immersion will not be the goal of every haptician, but the inclusion of haptics is valuable for virtual reality settings for producing a more involved and believable experience.

Expressivity means to feel the haptics distinguishably reflect varying user input and system events. While not a requirement in all haptic systems as singular haptic effects can still be impactful, expressivity allows users to feel their input make an impact on the feedback. Expanding the repertoire of effects offered to the users can also increases the information that can be transferred to users through touch.

Autotelics is an additional factor recommended by HE1 that was not reflected in the card sort task. Autotelics is a purely hedonic-driven dimension, referring to the touch experience as an end in and of itself [35]. Users may describe their experience as positive even when interacting with contextfree haptics. An effect may not exceed expectation on the side of functionality but can contribute to the experience by simply feeling pleasurable in and of itself.

\section{Personalization: Customizing HX}

There exist individual differences in the perception and preference of touch $[38,39]$. Therefore, benefiting the user experience is the option to adjust the haptic settings to match user preferences, either through changing elements of the design parameters or turning them off altogether [45]. Novices supported personalization through the lens of accessibility (a term modified by HE5 to reflect the idea of tunability) along with experts HE1, HE3, and HE4. HE1 articulated the importance of granting users control having found that "if they are in control and they know they're in control early in the process, they have a better experience."

\section{DISCUSSION}

By articulating usability and experiential factors, the HX model can support researchers, designers, hapticians, and haptic users understand, communicate, and evaluate haptics.

\section{Implications for interaction and experience designers}

These factors can organize thinking about design for haptic technology. They might serve as the basis for a structured approach to haptic design training, an industry need stated by HE1. With more development, they could help further develop the discipline of haptic experience design, going beyond existing practices. [41]. These factors can be used as a universal set of design criteria that can lead to better links and collaborations between hapticians and other designers.

\section{Divergence from UX}

Developing these factors gives us a theoretical structure for understanding the differences between HX and UX. The sense of touch is multimodal and haptic devices are stunningly diverse in their attempts to support it. This complexity, with a lack of language or other cognitive 
support, distinguishes HX from other experiences with technology. This aligns with our findings of multi-modal integration (harmony) being the most important experiential quality in the model, in addition to the inclusion of realism and immersion, two factors that are largely multimodal. HX rests heavily on the ability to achieve cohesion between the haptics and the remaining system components.

The resulting usability requirements also diverged from traditional usability heuristics. For instance, visibility of system status is a standard interaction design principle [49], but was not mentioned as a focus by our participants. Common usability criteria have largely been sight-focused; therefore, to design experience for a different sense, i.e. touch, usability requirements should reflect the shift in focus.

\section{Implications for researchers}

The HX model can guide future research. By formulating these factors and looking at the pairwise relationships between them, we found several gaps in the literature. For example, the relationship between Harmony and Immersion: a system can offer a harmonious system without being immersive (e.g., a wearable device offering haptic feedback that aligns with other sensory modalities, but the device is intended for low-attention information display and not for immersive experiences), but it unknown whether immersion could achieved in the absence of harmony. Researchers may test the effects of harmony on immersion, or make similar comparisons with alternative factor pairs, to reveal the influence of individual factors. By doing so, it would reveal whether certain factors serve as prerequisites to others insight valuable for both researchers and designers.

\section{Implications for Evaluation}

Our findings underscore the lack of evaluation tools for haptic design [41]: hapticians need a way to vouch for their haptics and identify areas for improvement. Currently, haptics systems are evaluated laboriously and independently, and hapticians have noted the absence of a widely available metric specific to their field. This model is the first step towards informing a Haptic Experience Index (HXI), a tool aimed at assessing the haptic component of a system. Having a universal and consistent metric that captures the factors of the HX model will serve hapticians and their systems by highlighting strengths, diagnosing deficiencies, enabling comparisons between systems, and allowing for a simple means of communication between user and designer.

\section{Bridging hapticians and novices with new language}

One major barrier facing the adoption of haptic technology and understanding of $\mathrm{HX}$ is the lack of an established language [25], especially for those unfamiliar with haptics [41]. As indicated by HE1, minimal familiarity with haptics makes communicating about HX difficult, creating challenges for hapticians and designers when requesting feedback from users. HE2 also came across a similar problem with users: "it feels good...I don't know why it feels good" (HE2). The HX model is a start to bridging the gap between haptic novices and experts by giving those new to haptics the vocabulary to describe and elaborate what they experience. The model includes elements produced largely by those with low haptic familiarity, represented by terms refined by those with extensive field expertise. Therefore, both novices and hapticians can easily detect and understand the factors offered by the model.

\section{Future Work: Creating a Universal HX Model}

Our work focused largely on vibrotactile feedback, particularly when generating responses from novice participants. However, vibrotactile feedback is a common and mature technology, making it an appropriate initial experience to offer for the critical step of probing novice input. Additionally, the expert hapticians were recruited to achieve diversity, including the modalities with which they typically work. We began development of the model using vibrotactile stimuli, then refined with insight from hapticians whose expertise ranged in modality. Experts offered some generalization through the nature of the haptics they discussed. While novices informed the model through their evaluation of existing haptic feedback experiences, experts also discussed those that are in active development or may occur in the future. Therefore, the model contains elements considered desirable for general HX, and is not necessarily limited only to existing systems. As result, we propose the current model as an initial structure, forming the foundation that, in future iterations, can be generalized to formally include several haptic modalities.

\section{CONCLUSION}

We report results produced in two workshops with novices and refined by six hapticians through interviews and a card sorting task. We define Haptic Experience (HX) and present a model representing the factors of $\mathrm{HX}$, informed by literature, haptic novices, and hapticians. The proposed HX model is characterized by four key components: Design Parameters, Usability Requirements, Experiential Dimensions, and Personalization.

We highlighted the current methods used and barriers faced by hapticians when evaluating user experience for haptic systems, and outlined the implications of the HX model in action for researchers, designers, hapticians, and end-users. We hope to see the model aid the understanding, communication, and evaluation of $\mathrm{HX}$.

\section{ACKNOWLEDGEMENTS}

We thank Lennart Nacke for advice on the scale development process, the University of Waterloo HCI community for their continuous guidance, Raj Shah for organizational assistance, and our participants for their input.

We acknowledge the support of the Natural Sciences and Engineering Research Council of Canada (NSERC), [funding reference number 2019-06589].

Cette recherche a été financée par le Conseil de recherches en sciences naturelles et en génie du Canada (CRSNG), [numéro de référence 2019-06589]. 


\section{REFERENCES}

[1] Azmandian, M. et al. 2016. Haptic Retargeting: Dynamic repurposing of passive haptics for enhanced virtual reality experiences. Proceedings of the 2016 CHI Conference on Human Factors in Computing Systems - CHI '16 (New York, New York, USA, 2016), 1968-1979.

[2] Bargas-Avila, J.A. and Hornbæk, K. 2011. Old wine in new bottles or novel challenges. Proceedings of the 2011 annual conference on Human factors in computing systems - CHI' 11 (New York, New York, USA, May 2011), 2689.

[3] Bau, O. et al. 2010. TeslaTouch: Electrovibration for Touch Surfaces. Proceedings of the 23nd annual ACM symposium on User interface software and technology UIST '10 (New York, New York, USA, Oct. 2010), 283.

[4] Berger, C.C. et al. 2018. The uncanny valley of haptics. Science Robotics. 3, 17 (2018), Art--No.

[5] Best HD Rumble games for Nintendo Switch that aren't 1-2 Switch: 2018. https://www.imore.com/best-hdrumble-games-arent-1-2-switch.

[6] Boateng, G.O. et al. 2018. Best practices for developing and validating scales for health, social, and behavioral research: a primer. Frontiers in public health. 6, (2018).

[7] Bradley, A. et al. 2015. Gendered or neutral?: considering the language of HCI. Proceedings of the 41st Graphics Interface Conference (2015), 163-170.

[8] Brave, S. and Dahley, A. 1997. inTouch: a medium for haptic interpersonal communication. $\mathrm{CHI}$ '97 extended abstracts on Human factors in computing systems looking to the future - CHI '97 (New York, NY, USA, 1997), 363-364.

[9] Brockmyer, J.H. et al. 2009. The development of the Game Engagement Questionnaire: A measure of engagement in video game-playing. Journal of Experimental Social Psychology. 45, 4 (2009), 624634.

[10] Bucci, P. et al. 2018. Is it Happy?: Behavioural and Narrative Frame Complexity Impact Perceptions of a Simple Furry Robot's Emotions. Proceedings of the 2018 CHI Conference on Human Factors in Computing Systems (2018), 509.

[11] Cang, X.L. et al. 2015. Different Strokes and Different Folks. Proceedings of the 2015 ACM on International Conference on Multimodal Interaction - ICMI '15 (New York, New York, USA, 2015), 147-154.

[12] Carter, T. et al. 2013. UltraHaptics: multi-point mid-air haptic feedback for touch surfaces. Proceedings of the 26th. (2013).
[13] Crawford, J.R. and Henry, J.D. 2004. The Positive and Negative Affect Schedule (PANAS): Construct validity, measurement properties and normative data in a large non-clinical sample. British journal of clinical psychology. 43, 3 (2004), 245-265.

[14] DeVellis, R.F. 2016. Scale development: Theory and applications. Sage publications.

[15] Doizaki, R. et al. 2017. Automatic Estimation of Multidimensional Ratings from a Single SoundSymbolic Word and Word-Based Visualization of Tactile Perceptual Space. IEEE Transactions on Haptics. 10, 2 (2017), 173-182. DOI:https://doi.org/10.1109/TOH.2016.2615923.

[16] Feygin, D. et al. 2002. Haptic guidance: experimental evaluation of a haptic training method for a perceptual motor skill. Proceedings 10th Symposium on Haptic Interfaces for Virtual Environment and Teleoperator Systems. HAPTICS 2002 (2002), 40-47.

[17] Flagg, A. and MacLean, K. 2013. Affective touch gesture recognition for a furry zoomorphic machine. Proceedings of the 7th International Conference on Tangible, Embedded and Embodied Interaction - TEI '13 (New York, New York, USA, 2013), 25.

[18] Hamam, A. et al. 2008. A quality of experience model for haptic user interfaces. Proceedings of the 2008 Ambi-Sys workshop on Haptic user interfaces in ambient media systems (2008), 1.

[19] Hassenzahl, M. et al. 2003. AttrakDiff: Ein Fragebogen zur Messung wahrgenommener hedonischer und pragmatischer Qualität. Vieweg+Teubner Verlag. 187196.

[20] Hassenzahl, M. et al. 2010. Needs, affect, and interactive products - Facets of user experience. Interacting with Computers. 22, 5 (Sep. 2010), 353362. DOI:https://doi.org/10.1016/j.intcom.2010.04.002.

[21] Hassenzahl, M. 2007. The hedonic/pragmatic model of user experience. Towards a UX manifesto. 10, (2007).

[22] Hoggan, E. et al. 2008. Crossmodal congruence: The look, feel and sound of touchscreen widgets. ICMI'08: Proceedings of the 10th International Conference on Multimodal Interfaces. (2008), 157-164. DOI:https://doi.org/10.1145/1452392.1452423.

[23] Hollins, M. et al. 2000. Individual differences in perceptual space for tactile textures: Evidence from multidimensional scaling. Perception \& Psychophysics. 62, 8 (Dec. 2000), 1534-1544. DOI:https://doi.org/10.3758/BF03212154.

[24] How the Nintendo Switch's HD rumble makes Tumbleseed feel real: 2017. https://www.polygon.com/2017/5/1/15499328/tumblese ed-hd-rumble-nintendo-switch. 
[25] Jansson-Boyd, C. V. 2011. Touch matters: exploring the relationship between consumption and tactile interaction. Social Semiotics. 21, 4 (Sep. 2011), 531546.

DOI:https://doi.org/10.1080/10350330.2011.591996.

[26] Karuei, I. et al. 2011. Detecting vibrations across the body in mobile contexts. Proceedings of the 2011 annual conference on Human factors in computing systems - CHI '11 (New York, New York, USA, 2011), 3267.

[27] Klatzky, R.L. and Peck, J. 2012. Please touch: Object properties that invite touch. IEEE Transactions on Haptics. 5, 2 (2012), 139-147. DOI:https://doi.org/10.1109/TOH.2011.54.

[28] Laugwitz, B. et al. 2008. Construction and evaluation of a user experience questionnaire. Symposium of the Austrian HCI and Usability Engineering Group (2008), 63-76.

[29] Law, E.L.-C. et al. 2018. Systematic Review and Validation of the Game Experience Questionnaire (GEQ)-Implications for Citation and Reporting Practice. Proceedings of the 2018 Annual Symposium on Computer-Human Interaction in Play (2018), 257270.

[30] Law, E.L.-C. et al. 2009. Understanding, scoping and defining user experience. Proceedings of the $27 \mathrm{th}$ international conference on Human factors in computing systems - CHI 09 (New York, New York, USA, Apr. 2009), 719.

[31] Maggioni, E. et al. 2017. Measuring the added value of haptic feedback. 2017 9th International Conference on Quality of Multimedia Experience, QoMEX 2017. (2017), 1-6.

DOI:https://doi.org/10.1109/QoMEX.2017.7965670.

[32] Minge, M. et al. 2016. Developing and validating an English version of the meCUE questionnaire for measuring user experience. Proceedings of the Human Factors and Ergonomics Society Annual Meeting (2016), 2063-2067.

[33] Nagano, H. et al. 2014. Haptic Invitation of Textures: Perceptually Prominent Properties of Materials Determine Human Touch Motions. IEEE Transactions on Haptics. (2014), 1-1.

DOI:https://doi.org/10.1109/TOH.2014.2321575.

[34] Nintendo Switch review: the BigN knocks it out of the park: 2019. https://www.t3.com/reviews/nintendoswitch-review.

[35] Obrist, M. et al. 2015. Emotions Mediated Through Mid-Air Haptics. Proceedings of the 33rd Annual ACM Conference on Human Factors in Computing Systems CHI ' 15 (New York, New York, USA, Apr. 2015), 2053-2062.
[36] Obrist, M. et al. 2013. Talking about tactile experiences. Proceedings of the SIGCHI Conference on Human Factors in Computing Systems (CHI'13) (New York, USA, 2013), 1659-1668.

[37] Okamoto, S. et al. 2013. Psychophysical Dimensions of Tactile Perception of Textures. IEEE Transactions on Haptics. 6, 1 (Jan. 2013), 81-93. DOI:https://doi.org/10.1109/TOH.2012.32.

[38] Peck, J. and Childers, T.L. 2003. Individual Differences in Haptic Information Processing: The "Need for Touch" Scale. Journal of Consumer Research. 30, 3 (2003), 430-442. DOI:https://doi.org/10.1086/378619.

[39] Peck, J. and Wiggins, J. 2006. It Just Feels Good: Customers' Affective Response to Touch and Its Influence on Persuasion. Journal of Marketing. 70, 4 (2006), 56-69. DOI:https://doi.org/10.1509/jmkg.70.4.56.

[40] Russell, J.A. 1980. A circumplex model of affect. Journal of Personality and Social Psychology. 39, 6 (1980), 1161-1178. DOI:https://doi.org/10.1037/h0077714.

[41] Schneider, O. et al. 2017. Haptic experience design: What hapticians do and where they need help. International Journal of Human Computer Studies. 107, August 2016 (2017), 5-21. DOI:https://doi.org/10.1016/j.ijhcs.2017.04.004.

[42] Schneider, O.S. and MacLean, K.E. 2014. Improvising Design with a Haptic Instrument. HAPTICS '14 (Houston, USA, 2014).

[43] Schrepp, M. et al. 2014. Applying the user experience questionnaire (UEQ) in different evaluation scenarios. International Conference of Design, User Experience, and Usability (2014), 383-392.

[44] Sefidgar, Y.S. et al. 2016. Design and Evaluation of a Touch-Centered Calming Interaction with a Social Robot. IEEE Transactions on Affective Computing. 7, 2 (Apr. 2016), 108-121. DOI:https://doi.org/10.1109/TAFFC.2015.2457893.

[45] Seifi, H. 2019. Personalizing Haptics: From Individuals' Sense-Making Schemas to End-User Haptic Tools. Springer.

[46] Seifi, H. et al. 2015. VibViz: Organizing, visualizing and navigating vibration libraries. World Haptics ' 15 (2015).

[47] Seifi, H. and MacLean, K.E. 2013. A first look at individuals' affective ratings of vibrations. World Haptics Conference (Apr. 2013), 605-610.

[48] Seifi, H. and MacLean, K.E. 2017. Exploiting haptic facets: Users' sensemaking schemas as a path to design and personalization of experience. International Journal of Human-Computer Studies. 107, (2017), 3861. 
[49] Sharp, H. et al. Interaction Design: Beyond humancomputer interaction.

[50] Sheridan, T.B. 1992. Musings on telepresence and virtual presence. Presence: Teleoperators \& Virtual Environments. 1, 1 (1992), 120-126.

[51] Tondello, G.F. et al. 2019. "I don't fit into a single type": A Trait Model and Scale of Game Playing Preferences. IFIP Conference on Human-Computer Interaction (2019), 375-395.

[52] User Interface Design GmbH AttrakDiff.

[53] Watanabe, J. et al. 2012. Visualization of Tactile Material Relationships Using Sound Symbolic Words.

Haptics: Perception, Devices, Mobility, and Communication. P. Isokoski and J. Springare, eds. Springer. 175-180.

[54] Watson, D. and Clark, L.A. 1999. The PANAS-X: Manual for the positive and negative affect scheduleexpanded form. (1999).

[55] Winfield, L. et al. 2007. T-PaD: Tactile Pattern Display through Variable Friction Reduction. Second Joint
EuroHaptics Conference and Symposium on Haptic Interfaces for Virtual Environment and Teleoperator Systems (WHC'07) (Mar. 2007), 421-426.

[56] Witmer, B.G. and Singer, M.J. 1998. Measuring Presence in Virtual Environments: A Presence Questionnaire. Presence. 7, 3 (1998), 225-240. DOI:https://doi.org/10.1117/12.2233447.

[57] Yohanan, S. et al. 2005. Hapticat: exploration of affective touch. Proceedings of the 7th international conference on Multimodal interfaces (New York, NY, USA, 2005), 222-229.

[58] Yongjae Yoo et al. 2015. Emotional responses of tactile icons: Effects of amplitude, frequency, duration, and envelope. 2015 IEEE World Haptics Conference (WHC) (Jun. 2015), 235-240.

[59] Yoo, T. et al. 2014. An Explorative Study on Crossmodal Congruence Between Visual and Tactile Icons Based on Emotional Responses. Proceedings of the 16th International Conference on Multimodal Interaction - ICMI '14 (New York, New York, USA, Nov. 2014), 96-103. 